\title{
Molecular response to the pathogen Phytophthora sojae among ten soybean near isogenic lines revealed by comparative transcriptomics
}

Feng Lin ${ }^{1 \dagger}$, Meixia Zhao ${ }^{1 \dagger}$, Douglas D Baumann², Jieqing Ping ${ }^{1}$, Lianjun Sun ${ }^{1}$, Yunfeng Liu', Biao Zhang ${ }^{1}$, Zongxiang Tang ${ }^{1}$, Elisa Hughes ${ }^{1}$, Rebecca W Doerge ${ }^{3}$, Teresa J Hughes ${ }^{4,5^{*}}$ and Jianxin Ma ${ }^{1 *}$

\begin{abstract}
Background: Phytophthora root and stem rot (PRR) of soybean, caused by Phytophthora sojae, is controlled by Rps genes. However, little is known regarding the Rps-induced molecular responses to P. sojae and how they actually overlap. We thus sequenced, analyzed, and compared the transcriptomes of 10 near isogenic lines (NILs), each with a unique Rps gene/allele, and the susceptible parent Williams, pre- and post-inoculation with the pathogen.

Results: A total of 4,330 differentially expressed genes (DEGs) were identified in Williams versus 2,014 to 5,499 DEGs in individual NILs upon inoculation with the pathogen. Comparisons of the DEGs between the NILs and Williams identified incompatible interaction genes (IIGs) and compatible interaction genes (CIGs). Hierarchical cluster and heatmap analyses consistently grouped the NILs into three clusters: Cluster I (Rps 1-a), Cluster II (Rps 1-b, 1-c and 1-k) and Cluster III (Rps3-a, 3-b, 3-c, 4, 5, and 6), suggesting an overlap in Rps-induced defense signaling among certain NILs. Gene ontology (GO) analysis revealed associations between members of the WRKY family and incompatible reactions and between a number of phytohormone signaling pathways and incompatible/compatible interactions. These associations appear to be distinguished according to the NIL clusters.

Conclusions: This study characterized genes and multiple branches of putative regulatory networks associated with resistance to $P$. sojae in ten soybean NILs, and depicted functional "fingerprints" of individual Rps-mediated resistance responses through comparative transcriptomic analysis. Of particular interest are dramatic variations of detected DEGs, putatively involved in ethylene (ET)-, jasmonic acid (JA)-, (reactive oxygen species) ROS-, and (MAP-kinase) MAPK- signaling, among these soybean NILs, implicating their important roles of these signaling in differentiating molecular defense responses. We hypothesize that different timing and robustness in defense signaling to the same pathogen may be largely responsible for such variations.
\end{abstract}

Keywords: Comparative transcriptomics, Resistance to Phytophthora sojae, Soybean

\footnotetext{
*Correspondence: hughestj@purdue.edu; maj@purdue.edu

${ }^{\dagger}$ Equal contributors

${ }^{4}$ Department of Botany and Plant Pathology, Purdue University, West Lafayette, IN 47907, USA

'Department of Agronomy, Purdue University, West Lafayette, IN 47907, USA

Full list of author information is available at the end of the article
} waiver (http://creativecommons.org/publicdomain/zero/1.0/) applies to the data made available in this article, unless otherwise stated. 


\section{Background}

Phytophthora root and stem rot (PRR) is one of the most devastating diseases of soybean (Glycine max), causing nearly $\$ 200$ million in annual yield losses in the U.S. alone [1]. PRR is caused by the soil-borne, hemibiotrophic pathogen Phytophthora sojae, and is most effectively controlled by Rps (Resistance to $P$. sojae) genes. The resistance conferred by an Rps gene is race specific, and the interaction between an Rps gene and the corresponding avirulence $(A v r)$ gene in $P$. sojae, follows the gene-for-gene model [2]. Currently, 18 Rps genes/alleles from soybean and $12 A v r$ genes from $P$. sojae have been identified [3-5].

Like most resistance $(R)$ genes, the $R p s$ gene family encodes, or is predicted to encode nucleotide binding leucine rich repeat (NB-LRR)-type proteins [6-8], which are able to recognize the $A v r$ effector proteins of pathogens directly or indirectly to induce the appropriate defense response $[9,10]$. The first evidence of a direct interaction between an $R$ and $A v r$ protein was reported in the flaxMelampsora lini pathosystem [11]. Indirect interactions, however, are more common. In these cases, the $R$ protein appears to require the existence of a 'guard protein' or 'decoy' in order to recognize an Avr protein [12,13]. A classical example of this type of interaction is the RPM1-interacting protein 4 (RIN4), which is 'guarded' by RPM1 (RESISTANCE TO PSEUDOMONAS SYRINGAE PV MACULICOLA 1) and RPS2 (RESISTANCE TO P. SYRINGAE 2) proteins in Arabidopsis [14,15].

The recognition of pathogen effectors triggers an innate immunity response that is mediated by two distinct types of NB-LRR proteins, the toll-interleukin-1 receptor (TIR)-NB-LRR proteins and the coiled-coil (CC)-NBLRR proteins. The former requires EDS1 (ENHANCED DISEASE SUSCEPTIBILITY 1), a central regulator of effector-triggered immunity (ETI), which functions together with PAD4 (PHYTOALEXIN DEFICIENT 4). In contrast, the latter NB-LRR proteins are independent of EDS1 but require NDR1 (NON-RACE-SPECIFIC DISEASE RESISTANCE 1) [16-21]. The interaction among these intracellular proteins results in a regulation network of phytohormone signaling, which is mainly mediated by salicylic acid (SA) for biotrophic and hemibiotrophic pathogens, and jasmonic acid (JA) and ethylene (ET) for necrotrophic pathogens $[8,22]$. In addition to SA, JA and ET, other phytohormones such as brassinosteroids (BR), abscisic acid (ABA), auxin, gibberellins (GA) and cytokinin (CK) also contribute to plant immune responses, with complex crosstalk between one another [23].

It is suggested that the resistance to $P$. sojae conferred by Rps genes is mediated by the SA signaling pathway, with the induction of SA-mediated systemic acquired resistance (SAR) occurring several days post infection (dpi) via expression of the gene NPR1 [24-26]. During this process, two putative regulators of the chromosome condensation 1 (RCC1) gene family are down-regulated during the incompatible interaction [27]. Besides SA, exogenous treatment of 1-aminocyclopropane-1-carboxylic acid (ACC, a precursor of ET) has been shown to enhance resistance while applications of GA and ABA induce susceptibility, highlighting the complexity of phytohormone signaling pathways in response to attack by $P$. sojae $[26,28,29]$.

A microarray study of transcriptomes from one susceptible and two partially resistant soybean genotypes indicated that $97-99 \%$ of all detectable genes experienced transcriptional modulation five dpi in response to infection by $P$. sojae [30]. However, the majority of these differences were less than two fold. Another microarray study of transcriptomic changes in soybean revealed a peak in most defense related genes at 24 hours post inoculation (hpi) with P. sojae [24]. Recently, Zhang et al conducted a proteomics study in which 46 differentially expressed proteins were identified in soybean after infection with P. sojae [31]. Among these, 26 were affected during the incompatible interaction, while the other 20 were altered during the compatible interaction. These studies have contributed to our understanding of the interaction between soybean and P. sojae. Nevertheless, what mechanisms underlie compatible and incompatible interactions, how molecular responses mediated by a variety of Rps genes/alleles resemble or differ from one another, as well as the nature of these similarity or difference, remain largely unknown.

Access to the soybean genome sequence of Williams 82 (Rps1-k) [32], and the advent of high-throughput RNA sequencing (RNA-Seq) by next-generation sequencing technology, have allowed researchers to take a novel look at the molecular interaction between soybean and P. sojae. Kim et al reported the first application of RNA-Seq for profiling gene expression in soybean in response to pathogen attack [33]. In this study, the transcriptomes of two near isogenic lines (NILs), one resistant and one susceptible to bacterial leaf pustule, were analyzed 0,6 , and $12 \mathrm{hpi}$ and a total of 2,761 differentially expressed genes (DEGs), including a set of defense response genes, were identified.

NILs are pure breeding lines that are developed by backcrossing a donor line with the recurrent parent for at least five generations to achieve introgression of a desired trait. As such, they share more than 98\% genetic identity with the recurrent parent, except for the region where the desired gene is located. In order to determine the molecular mechanisms responsible for Rps-mediated defense and to understand molecular basis for diverse responses to the pathogen, we analyzed the transcriptomes of 10 NILs, each with a unique Rps gene/allele, along with the susceptible recurrent parent Williams, pre- and post-inoculation with a race 1 isolate of $P$. sojae (avirulent towards NILs; virulent towards Williams). 


\section{Results}

Symptom development in $10 \mathrm{NILs}$ and the recurrent susceptible parent inoculated with $P$. sojae

The 10 soybean NILs carrying Rps1-a, Rps1-b, Rps1-c, Rps1-k, Rps3-a, Rps3-b, Rps3-c, Rps4, Rps5, or Rps6, within the Williams background, provided a unique resource for investigating the common and specific defense responses mediated by individual Rps genes against $P$. sojae (Additional file 1). Phenotypic reactions of the 10 NILs and Williams to $P$. sojae 7 days post-inoculation (dpi) with the pathogen were evaluated under greenhouse conditions. In each of three experimental replications, approximately half the number of seedlings from each line was inoculated with race 1 of $P$. sojae. The remaining seedlings were "mock" inoculated in the same manner without the pathogen. In each experimental replicate, Williams was susceptible (expanding lesion/plant death) to $P$. sojae, while all NILs were resistant (Additional file 2). NILs containing Rps1-a, Rps1-b, Rps1-c, Rps1-k and Rps4 displayed $100 \%$ survival when challenged with the pathogen. In contrast, NILs containing Rps3-a, Rps3-b, Rps3-c, Rps5 and Rps6 showed a slight variation in the proportion of surviving seedlings across the three replicates, which may be attributed to minor differences in environmental conditions. Despite this variation, the percentage of surviving seedlings in each replicate was equal to or greater than $75 \%$, which is generally used as a criterion for defining a pure line resistance [34]. All mock-inoculated seedlings were asymptomatic of PRR (Additional file 2).

\section{Transcriptional changes in 10 NILs and the recurrent susceptible parent in response to $P$. sojae}

RNAs, representing the entire transcriptomes of $P$. sojae inoculated and mock-inoculated seedlings from each of the 10 NILs and Williams 24 hpi with the pathogen, were sequenced and 14.5 to 50.1 million raw reads were generated for individual samples (Table 1). Trimming adaptor sequences and elimination of low quality reads and reads shorter than $101 \mathrm{bp}$, resulted in 13.5 to 39.5 million clean reads for each sample. Among these, $71.5 \%$ to $87.8 \%$ per sample were uniquely mapped to the soybean reference genome v1.0 (Table 1 ).

Based on the 46,367 high-confidence gene models annotated in the soybean reference genome [32], the relative change in the abundance of reads mapped to the same genes for inoculated and mock-treated samples, and the criteria for defining DEGs pre- and post-inoculation, a total of 9,847 non-redundant gene models were identified as DEGs in at least one of the 11 soybean lines. The numbers of DEGs identified between Williams and the 10 NILs or among the 10 NILs vary greatly. For example, 2,014 to 2,366 DEGs were found in individual NILs containing Rps1-a, Rps1-b, Rps1-c, or Rps1-k, while 3,038 to 5,499 DEGs were detected in individual NILs containing Rps3- $a$,
Rps3-b, Rps3-c, Rps4, Rps5 or Rps6 (Table 2). Among the 10 NILs, 1,274 to 2,823 DEGs were up-regulated, while 643 to 2,744 DEGs were down-regulated in response to inoculation with the pathogen (Table 2). In comparison, 2,460 up-regulated and 1,870 down-regulated DEGs were identified in Williams upon inoculation with P. sojae.

To validate DEGs profiled by RNA-Seq analysis, six detected DEGs per soybean line (Additional file 3) were randomly chosen for quantitative RT-PCR (qRT-PCR) analysis with the same RNA samples as used for RNA-Seq. Subsequently, the patterns of differential expression of these genes detected by RNA-Seq and qRT-PCR were compared. As shown in Additional file 4, significant correlations between the patterns of DEGs detected by the two approaches were observed for each set of genes chosen from 11 individual lines (Pearson's correlation coefficient ( $\mathrm{r}$ ), ranging from 0.725 to 0.994 ), indicating the reliability of DEGs identified by RNA-Seq (Additional file 4).

\section{Characterization of genes associated with incompatible, compatible, and basal interactions}

In an attempt to decipher the molecular basis of resistance and susceptibility to $P$. sojae, we applied a comparative transcriptomics approach to identify DEGs specifically associated with a host response. Three major groups of genes were classified: 1) Incompatible interaction genes (IIGs), are DEGs identified in NILs and associated with resistance; 2) Compatible interaction genes (CIGs) are DEGs identified in Williams and specifically associated with the susceptible response; and 3) Basal interaction genes (BIGs), which are DEGs shared by all NILs and Williams (Figure 1).

A total of 5,806 non-redundant IIGs, 1139 CIGs, and 835 BIGs were identified (Figure 1). The number of upregulated and down-regulated IIGs range from 159 to 1,158 and from 141 to 2,017 , respectively, among the 10 NILs. Of the 1,139 CIGs, 369 were up-regulated and 770 were down-regulated; and of the 835 BIGs, 696 were upregulated and 139 were down-regulated (Figure 1).

\section{Clusters of Rps-mediated IIGs}

To understand how transcriptomic changes mediated by different Rps genes/alleles overlap, we performed hierarchical cluster analysis using pvclust, an $\mathrm{R}$ package for assessing the uncertainty in hierarchical clustering [35], with $\log _{2}$ fold change (FC) of the 5,806 IIGs identified in at least one of the ten NILs. The NILs were grouped into three clusters, Clusters I, II, and III (Figure 2). Cluster I (C-I) consisted of the NIL containing Rps1-a only. Cluster II (C-II) was composed of those NILs containing Rps1-b, Rps1-c and Rps1-k, while Cluster III (C-III) included those NILs containing Rps3-a, Rps3-b, Rps3-c, Rps4, Rps5, and Rps6. This clustering of IIGs was further supported by the hierarchical cluster structure of the ten NILs revealed by 
Table 1 Statistics of pair-end reads in RNA sequencing experiment

\begin{tabular}{|c|c|c|c|c|}
\hline Soybean line & Raw reads & Clean reads $^{\mathrm{a}}$ & Uniquely mapped reads ${ }^{b}$ & $\%$ of uniquely mapped readsc \\
\hline \multicolumn{5}{|l|}{ Inoculated Group } \\
\hline Williams (rps) & $50,126,404$ & $39,475,154$ & $28,222,946$ & 71.5 \\
\hline Union (Rps1-a) & $25,614,448$ & $20,169,804$ & $14,969,954$ & 74.2 \\
\hline L77-1863 (Rps1-b) & $28,308,350$ & $22,460,578$ & $16,591,206$ & 73.9 \\
\hline L75-3735 (Rps1-C) & $19,696,090$ & $15,483,052$ & $11,084,896$ & 71.6 \\
\hline Williams82 (Rps1-k) & $14,508,392$ & $13,527,604$ & $11,527,676$ & 85.2 \\
\hline L83-570 (Rps3-a) & $21,982,116$ & $17,531,234$ & $12,954,567$ & 73.9 \\
\hline L91-8347 (Rps3-b) & $32,063,808$ & $25,210,242$ & $18,704,692$ & 74.2 \\
\hline L92-7857 (Rps3-c) & $29,003,280$ & $22,775,218$ & $16,776,075$ & 73.7 \\
\hline L85-2352 (Rps4) & $27,891,876$ & $21,857,446$ & $15,860,686$ & 72.6 \\
\hline L85-3059 (Rps5) & $35,204,260$ & $30,651,204$ & $24,816,154$ & 81.0 \\
\hline L89-1581 (Rps6) & $26,761,058$ & $23,224,142$ & $19,322,975$ & 83.2 \\
\hline \multicolumn{5}{|c|}{ Mock-inoculated Group } \\
\hline Williams (rps) & $21,949,578$ & $20,385,960$ & $17,841,238$ & 87.5 \\
\hline Union (Rps1-a) & $25,680,502$ & $23,860,934$ & $20,936,764$ & 87.7 \\
\hline L77-1863 (Rps1-b) & $20,303,214$ & $18,830,218$ & $16,453,627$ & 87.4 \\
\hline L75-3735 (Rps1-C) & $26,452,482$ & $22,841,526$ & $19,049,873$ & 83.4 \\
\hline Williams82 (Rps1-k) & $19,289,852$ & $16,845,090$ & $14,241,574$ & 84.5 \\
\hline L83-570 (Rps3-a) & $33,576,796$ & $29,006,366$ & $24,493,712$ & 84.4 \\
\hline L91-8347 (Rps3-b) & $25,609,764$ & $23,775,078$ & $20,880,124$ & 87.8 \\
\hline L92-7857 (Rps3-c) & $24,806,160$ & $22,983,728$ & $20,078,725$ & 87.4 \\
\hline L85-2352 (Rps4) & $24,677,426$ & $22,899,582$ & $20,085,744$ & 87.7 \\
\hline L85-3059 (Rps5) & $26,874,804$ & $23,304,436$ & $19,637,730$ & 84.3 \\
\hline L89-1581 (Rps6) & $27,363,314$ & $25,362,532$ & $22,151,262$ & 87.3 \\
\hline
\end{tabular}

${ }^{\mathrm{a}}$ Reads with a quality score less than 20 and length less than 101 nucleotides were excluded.

${ }^{\mathrm{b}}$ Reads uniquely mapped to G. max genome assembly (v1.0) using TopHat 2.0 software [58].

'Percentage of uniquely mapped reads out of clean reads.

heatmap analysis [36] (Additional file 5). In addition, the numbers of IIGs identified for each individual NIL, were unevenly distributed among different clusters (Figures 1 and 2). For example, 300, 1,193-3,073, and 556-614 IIGs were found in C-I, C-II, and C-III, respectively. The

Table 2 Differentially expressed genes (DEGs) in each soybean line

\begin{tabular}{lccc}
\hline Soybean line & $\begin{array}{c}\text { Up-regulated } \\
\text { DEGs }\end{array}$ & $\begin{array}{c}\text { Down-regulated } \\
\text { DEGs }\end{array}$ & Total \\
\hline Williams (rps) & 2460 & 1870 & 4330 \\
Union (Rps1-a) & 1432 & 643 & 2075 \\
L77-1863 (Rps1-b) & 1536 & 831 & 2366 \\
L75-3735 (Rps1-c) & 1532 & 698 & 2230 \\
Williams82 (Rps1-k) & 1274 & 740 & 2014 \\
L83-570 (Rps3-a) & 2362 & 1827 & 4189 \\
L91-8347 (Rps3-b) & 2823 & 2166 & 4989 \\
L92-7857 (Rps3-c) & 2749 & 2247 & 4996 \\
L85-2352 (Rps4) & 2470 & 2143 & 4613 \\
L85-3059 (Rps5) & 2755 & 2744 & 5499 \\
L89-1581 (Rps6) & 2033 & 1005 & 3038 \\
\hline
\end{tabular}

numbers of IIGs in NILs containing different alleles at a same gene locus also varied (Figures 1 and 2).

The IIGs among the three clusters were analyzed by both broad range comparison, where all the genes within a cluster were counted, and narrow range comparison, where only those genes shared by the NILs within a cluster were counted. Broad range comparison showed that 75 upregulated and 68 down-regulated IIGs were shared by the three clusters, whereas 62,214 , and 4,488 IIGs were unique to NILs in Clusters I, II, and III, respectively (Figure 3A,B). In contrast, narrow range comparison indicated that only 16 up-regulated and 7 down-regulated IIGs were shared among the three clusters (Figure 3C,D). The annotation of these 23 IIGs is listed in Table 3.

\section{Putative functions of DEGs based on gene ontology (GO) analysis}

To understand the functional components involved in molecular responses to $P$. sojae, we annotated the putative products encoded by IIGs, CIGs, and BIGs based on GO analysis (Figure 4; Additional file 6). These DEGs 


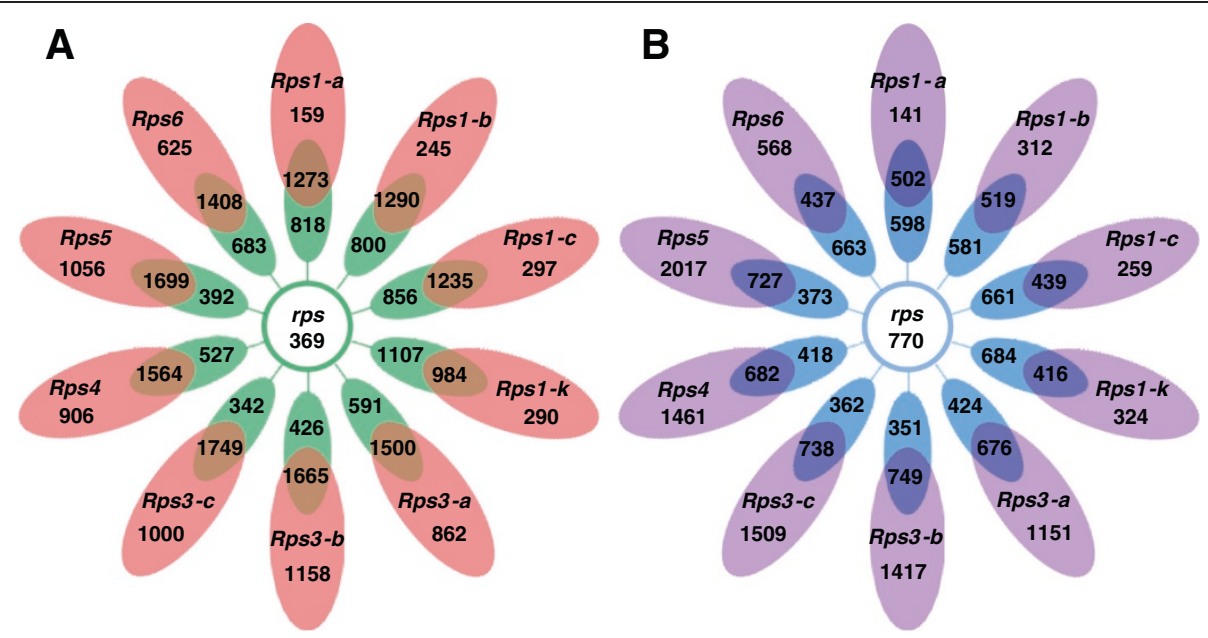

Figure 1 Comparison of differentially expressed genes (DEGs) between Williams (susceptible to Phytophthora sojae) and 10 near isogenic lines (NILs) each containing a single Rps (Resistance to $\boldsymbol{P}$. sojae) gene. A: up-regulated DEGs. B: down-regulated DEGs. Red and purple represent the number of DEGs specific to individual Rps genes and collectively referred to as incompatible interaction genes. Brown and dark purple represent the number of DEGs shared by an individual NIL and Williams. Green and light blue represent the number of DEGs specifically expressed in 'Williams' when compared to a specific NIL. The central green and light blue circles represent the common proportion of Williams specific DEGs that are collectively referred to as compatible interaction genes.

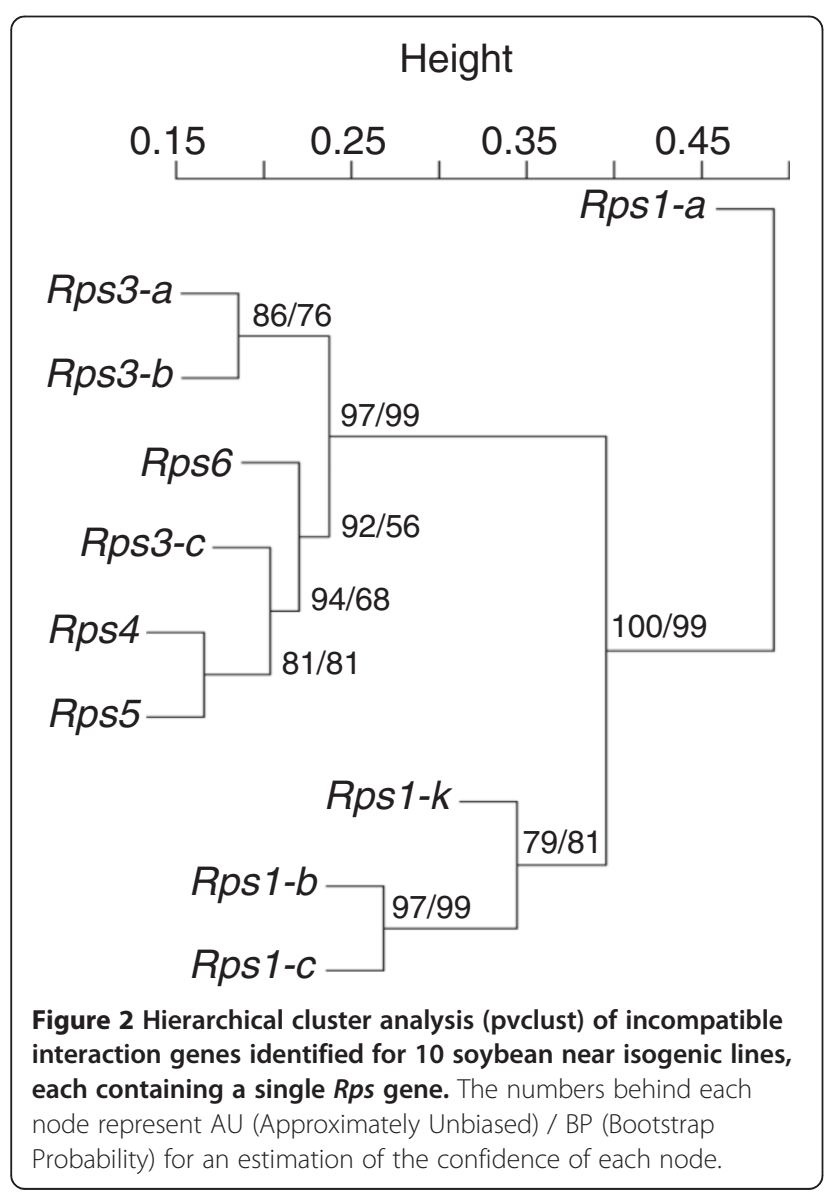

were grouped into seven categories: 1) Response to biotic or abiotic stress; 2) Biological regulation; 3) Growth and development; 4) Transport; 5) Metabolism; 6) Miscellaneous; and 7) Unclassified. For IIGs, the majority of annotated genes fell into the category of "response to biotic stress and abiotc stress" (Figure 4; Additional files 6 and 7). This pattern was also observed for CIGs and

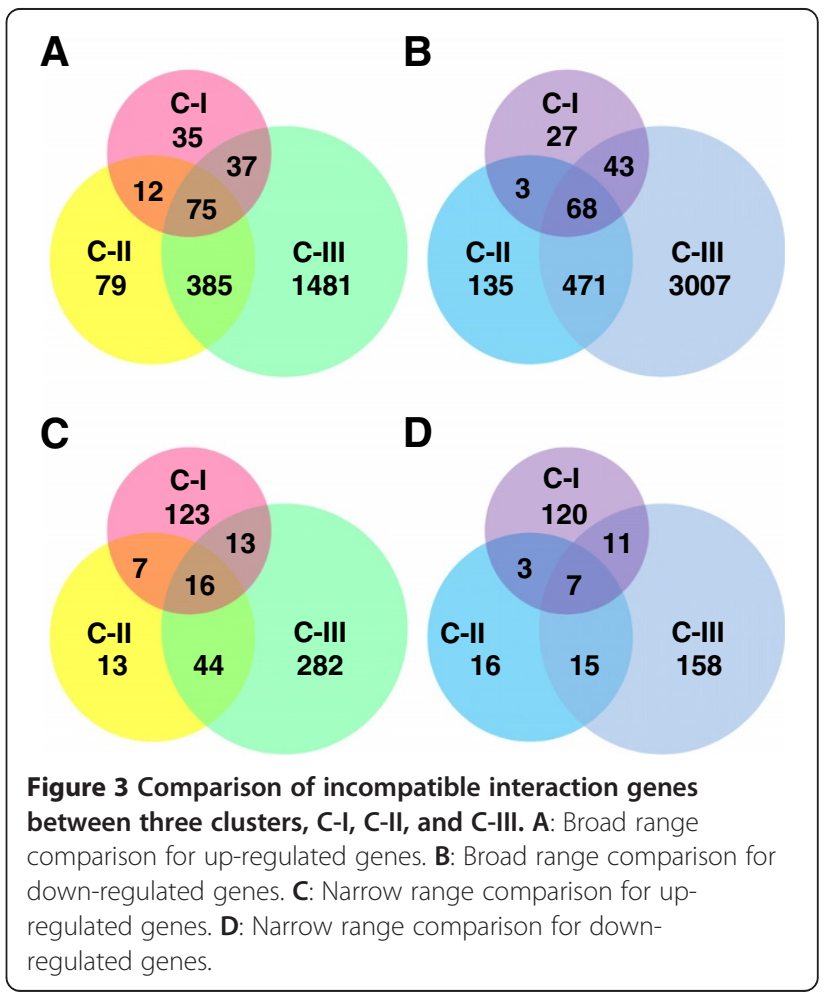


Table 3 Incompatible interaction genes (IIGs) shared by all the near isogenic lines and their annotation

\begin{tabular}{|c|c|c|c|c|}
\hline Gene & $\begin{array}{l}\text { Arabidopsis } \\
\text { homologous gene }\end{array}$ & Gene symbol & Gene annotation & GO group \\
\hline \multicolumn{5}{|l|}{ Up regulated } \\
\hline Glyma01g32130 & AT2G40435 & - & Transcription factor, bHLH & Response to biotic or abiotic stress \\
\hline Glyma02g16710 & AT1G03220 & - & Eukaryotic aspartyl protease family protein & Metabolism \\
\hline Glyma03g01010 & AT4G26010 & - & Peroxidase superfamily protein & Response to biotic or abiotic stress \\
\hline Glyma05g34870 & AT1G14870 & PCR2 & Plant cadmium resistance 2 & - \\
\hline Glyma06g09220 & AT5G25880 & NADP-ME3 & NADP-malic enzyme 3 & Response to biotic or abiotic stress \\
\hline Glyma06g13090 & AT5G24110 & WRKY30 & WRKY DNA-binding protein 30 & Response to biotic or abiotic stress \\
\hline Glyma09g15090 & AT4G21380 & RK3 & Receptor kinase 3 & Metabolism \\
\hline Glyma11g07430 & AT5G03260 & LAC11 & Laccase 11 & Metabolism \\
\hline Glyma13g17800 & AT4G04450 & WRKY42 & WRKY family transcription factor & Biological regulation \\
\hline Glyma13g30770 & AT1G28480 & GRX480 & Thioredoxin superfamily protein & Response to biotic or abiotic stress \\
\hline Glyma13g36070 & AT3G20660 & $4-\mathrm{Oct}$ & Organic cation/carnitine transporter 4 & Biological regulation \\
\hline Glyma14g39300 & AT1G66160 & CMPG1 & CYS, MET, PRO, and GLY protein 1 & Response to biotic or abiotic stress \\
\hline Glyma15g05810 & AT2G41480 & - & Peroxidase superfamily protein & Response to biotic or abiotic stress \\
\hline Glyma15g06010 & AT5G64260 & EXL2 & Exordium like 2 & - \\
\hline Glyma15g19600 & AT2G05940 & - & Protein kinase superfamily protein & Metabolism \\
\hline Glyma20g38000 & AT1G09090 & $\mathrm{RBOHB}$ & Respiratory burst oxidase homolog B & Response to biotic or abiotic stress \\
\hline \multicolumn{5}{|l|}{ Down regulated } \\
\hline Glyma04g12290 & AT1G71380 & CEL3 & Cellulase 3 & Response to biotic or abiotic stress \\
\hline Glyma09g37290 & AT5G15230 & GASA4 & GAST1 protein homolog 4 & Response to biotic or abiotic stress \\
\hline Glyma12g10670 & AT3G21700 & SGP2 & Ras-related small GTP-binding family protein & Response to biotic or abiotic stress \\
\hline Glyma12g29980 & AT4G39370 & - & Ubiquitin-specific protease 27 & - \\
\hline Glyma13g44210 & AT5G20190 & - & Tetratricopeptide repeat (TPR)-like superfamily protein & - \\
\hline Glyma15g18440 & AT4G29720 & $\mathrm{PAO5}$ & Polyamine oxidase 5 & Metabolism \\
\hline Glyma18g49400 & AT5G15230 & GASA4 & GAST1 protein homolog 4 & Response to biotic or abiotic stress \\
\hline
\end{tabular}

BIGs (Figure 4; Additional file 7). Of the 23 IIGs shared by the 10 NILs, 12, 2, and 5, were found to be related to "response to biotic or abiotic stress", "biological regulation", and "metabolism", respectively (Table 3). The remaining four shared genes could not be classified into any functional category.

\section{Putative transcription factors (TFs) involved in molecular responses to $P$. sojae}

It is documented that TFs play important roles in plant defense and stress responses. As such, we annotated and analyzed putative TFs in the sets of DEGs detected in this study based on a soybean TF database that is composed of 5,671 predicted TFs [37]. Of the 5,806 non-redundant IIGs, 282 up-regulated and 543 down-regulated genes were annotated as putative TFs. The up-regulated and downregulated IIG TFs range from 13 to 177 and from 21 to 307, respectively, among the 10 NILs (Additional files 8 and 9). The number of IIG TFs also varied among the three clusters, with 34, 164, and 791 in C-I, C-II, and C-III, respectively. The most abundant ones along these IIG TFs (either up-regulated or down-regulated) were of the WRKY family, accounting for $23.1 \%$ of all IIG TFs identified in C-I, 18.5-25.8\% in C-II, and 14.0-18.6\% in C-III (Additional file 9). Only three up-regulated IIG TFs (1 bHLH and 2 WRKY) and one down-regulated TF (TPR) were found to be shared by all the 10 NILs (Table 3; Additional file 9).

Of the 1,139 CIGs identified in Williams, 43 upregulated and 149 down-regulated genes were found to be putative TFs (Additional file 9). The largest proportion of up-regulated CIG TFs belonged to the MYB/HDlike family (16.3\%), followed by the AP2-EREBP family (14.0\%). In contrast, members of the AP2-EREBP family represented the largest number (20.8\%) of down-regulated CIG TFs, followed by MYB/HD-like (10.1\%) and bHLH (9.4\%). None of these 43 up-regulated CIG TFs belonged to WRKY family.

Of the 835 non-redundant BIGs shared by all the 11 soybean lines, 41 up-regulated and 9 down-regulated ones were found to be TFs (Additional file 9). Among 


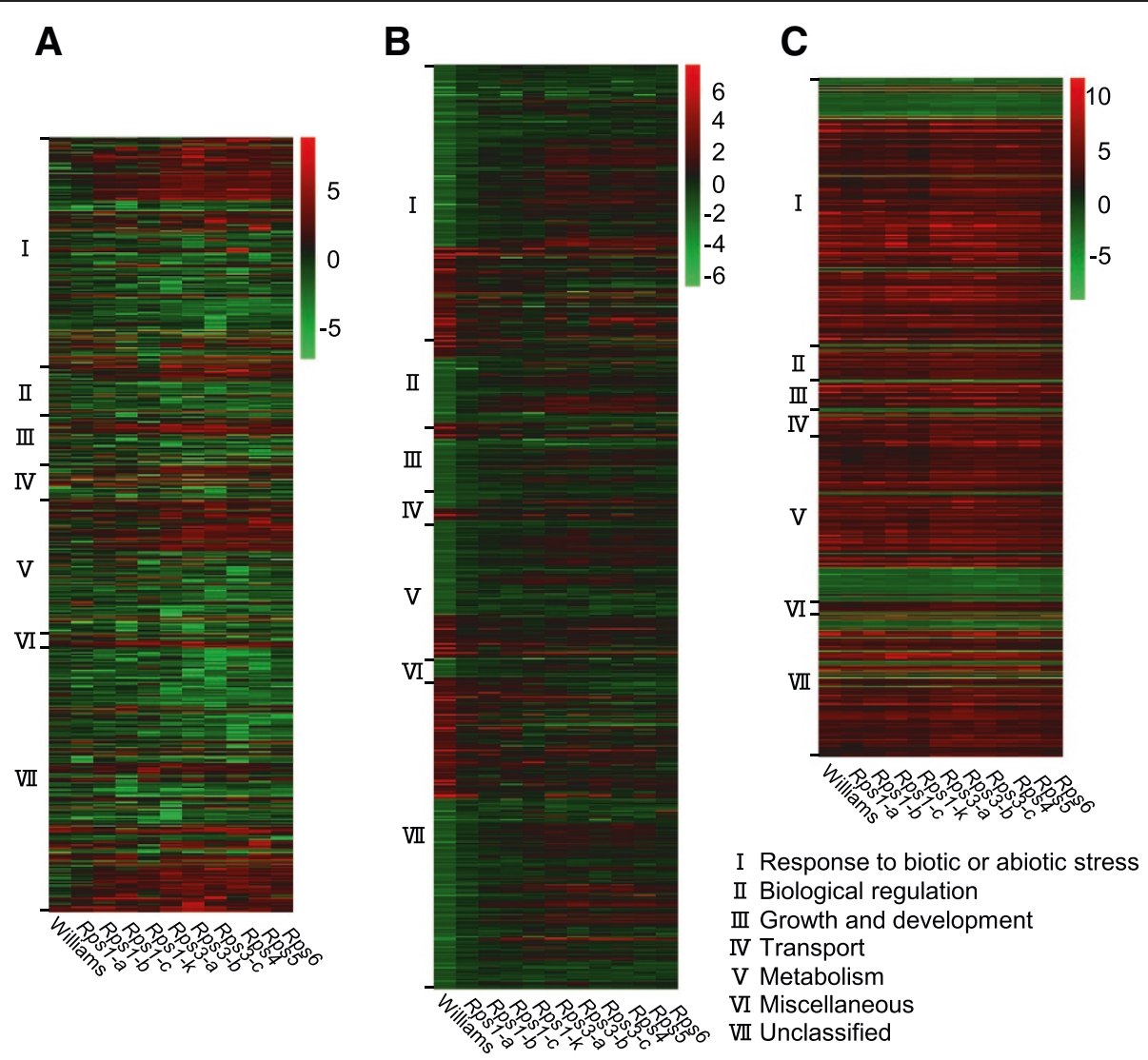

Figure 4 Heatmap analysis of three categories of differentially expressed genes based on gene ontology analysis. The values used to draw heatmaps are $\log _{2}$ (fold change) of expression level of post inoculation to mock inoculation. A: Incompatible interaction genes. The Log 2 (fold change) less than 3 in all 10 resistant near isogenic lines were not shown here. B: Compatible interaction genes. C: Core basal interaction genes.

the up-regulated BIG TFs, the most abundant ones are of the WRKY family (34\%), followed by the MYB/HDlike family (19.5\%).

\section{Knowledge-based comparative analysis of Rps gene-mediated defense signaling pathways}

The DEGs showing homologies to previously identified protein genes responsible for pathogen recognition and defense, or defense-related phytohormone signaling genes were further analyzed. Overall, these DEGs exhibited diverse patterns of distribution among the three NIL clusters (Figure 5). For example, of the 26 up-regulated IIGs homologous to previously reported defense/stress signaling genes, 24 were found only in NILs within Cluster III. In contrast, only one (Glyma20g38000) of the two $R B O H$ $B$ gene homologs was found to be up-regulated in all 10 NILs. It is notable that the majority of the putative DEGs involved in defense-related phytohormone signaling pathways showed distinct or opposite patterns of variation in gene expression between Williams and NILs, particularly the NILs within the Cluster III (Figure 5).

Previous studies indicated that the resistance to $P$. sojae in soybean was mediated by SA signaling [24-26], with the NPR1 as the key component of SA-mediated signaling [38]. In this study, two NPR1-like IIGs (Glyma02g45260 and Glyma14g03510) were identified (Figure 5). However, these two genes differ from the two NPR1-like genes reported by Sandhu et al [25]. In the later study, they studied soybean leaves four dpi with $P$. sojae race 4 while, in the present study, we studied stems $24 \mathrm{hpi}$ with $P$. sojae race 1 . As such, these two studies are less comparable.

As an antagonist of SA, the JA pathway is repressed by JAZ proteins in Arabidopsis [39,40]. Several JAZ homologs were identified as IIG and/or CIG, including homologs of JAZ1, JAZ6, JAZ8 and JAZ12 (Figure 5). These homologs showed a consistent expression pattern that was upregulated NILs and down-regulated in Williams. In contrast to the JAZ proteins, JAR1 (Jasmonate resistant1) appears to be required for the activation of the JA signaling pathway in Arabidopsis [41,42]. We found that a soybean JAR1 homolog (Glyma07g06370) was up-regulated in Williams upon inoculation with the P. sojae. These observations suggest that the JA signaling pathway may have played opposite roles in phytohormone signaling between incompatible and compatible interactions. 


\begin{tabular}{|c|c|c|c|c|c|c|c|c|c|c|c|c|c|}
\hline Gene_ID & $\begin{array}{c}\text { Gene } \\
\text { Symbol }\end{array}$ & Wms & $\begin{array}{c}\text { Rps1-a } \\
\text { C-1 }\end{array}$ & $\begin{array}{c}\text { Rps1-b } \\
\text { C-II }\end{array}$ & $\begin{array}{c}\text { Rps1-c } \\
\text { C-II }\end{array}$ & $\begin{array}{c}\text { Rps1-k } \\
\text { C-II }\end{array}$ & $\begin{array}{c}\text { Rps3-a } \\
\text { C-III }\end{array}$ & $\begin{array}{c}\text { Rps3-b } \\
\text { C-III }\end{array}$ & $\begin{array}{c}\text { Rps3-c } \\
\text { C-III }\end{array}$ & $\begin{array}{l}\text { Rps4 } \\
\text { C-III }\end{array}$ & $\begin{array}{l}\text { Rps5 } \\
\text { C-III }\end{array}$ & $\begin{array}{l}\text { Rps6 } \\
\text { C-IIII }\end{array}$ & $\begin{array}{l}\text { Gene } \\
\text { Group }\end{array}$ \\
\hline Glyma04g38700 & PAD4 & -0.79 & -0.59 & -0.69 & -0.66 & -0.78 & -1.29 & -0.93 & -1.08 & -1.32 & -1.25 & -1.08 & $\| G$ \\
\hline Glyma05g32760 & PAD4 & -1.27 & -0.48 & 0.12 & 0.04 & 0.53 & 0.91 & 0.91 & 0.68 & 1.04 & 1.13 & 0.91 & $\mathrm{CIG} / I \mathrm{GG}$ \\
\hline Glyma08g00420 & PAD4 & -0.47 & 0.11 & 0.23 & 0.19 & 0.66 & 1.21 & 1.17 & 1.16 & 1.17 & 1.41 & 1.08 & $\| G$ \\
\hline Glyma12g34210 & NDR1 & -0.49 & -0.43 & 0.39 & 0.62 & 0.06 & 0.84 & 1.45 & 0.87 & 0.76 & 1.02 & 1.05 & $\| G$ \\
\hline Glyma03g19920 & RIN4 & 0.74 & 0.67 & 0.46 & 0.59 & -0.01 & 1.21 & 0.99 & 1.50 & 1.22 & 1.42 & 0.94 & $\| G$ \\
\hline Glyma08g08810 & FLS2 & -0.95 & -0.11 & -0.45 & -0.56 & 0.48 & 0.86 & 1.23 & 2.09 & 1.16 & 1.23 & 1.66 & $\| G$ \\
\hline Glyma13g20800 & PAL & 0.90 & 0.51 & 0.61 & 0.63 & 0.46 & 0.76 & 0.81 & 1.08 & 1.01 & 1.19 & 1.01 & $\| G$ \\
\hline Glyma03g39610 & $\mathrm{RBOH} \mathrm{B}$ & -0.91 & -0.15 & 0.85 & 1.23 & 1.22 & 2.52 & 3.13 & 1.73 & 2.25 & 2.69 & 1.92 & $\| G$ \\
\hline Glyma20g38000 & $\mathrm{RBOH} \mathrm{B}$ & 0.93 & 1.40 & 1.48 & 1.95 & 1.74 & 1.83 & 2.11 & 2.27 & 1.78 & 1.65 & 1.42 & $\| G$ \\
\hline Glyma11g15700 & MPK3 & -0.52 & -0.18 & 0.26 & 0.22 & 0.29 & 1.33 & 1.75 & 1.14 & 1.44 & 1.64 & 1.10 & $\| G$ \\
\hline Glyma12g07770 & MPK3 & -0.40 & -0.10 & 0.36 & 0.54 & 0.44 & 1.50 & 1.99 & 1.45 & 1.37 & 1.86 & 1.36 & $\| G$ \\
\hline Glyma07g07270 & MPK4 & 0.68 & 0.62 & 1.45 & 1.31 & 0.45 & 2.01 & 2.11 & 2.15 & 2.23 & 2.39 & 2.09 & $\| G$ \\
\hline Glyma09g39190 & MPK4 & 0.93 & 0.43 & 0.59 & 0.10 & 0.17 & 0.59 & 0.86 & 1.48 & 0.93 & 1.19 & 1.20 & $\| G$ \\
\hline Glyma08g02060 & MPK4 & -0.46 & -0.80 & -0.03 & -1.78 & -5.77 & -1.25 & -0.39 & -0.83 & -1.74 & -1.80 & -0.34 & $\| G$ \\
\hline Glyma02g45260 & NPR1-like & 0.48 & 0.67 & 0.34 & 0.58 & 0.46 & 0.99 & 1.04 & 0.73 & 1.23 & 0.90 & 0.72 & IIG \\
\hline Glyma14g03510 & NPR1-like & 1.18 & 0.75 & 1.60 & 0.71 & 1.08 & 0.52 & 1.66 & 1.10 & -0.02 & 1.73 & 0.48 & $\| G$ \\
\hline Glyma09g08290 & JAZ1 & -0.84 & -0.48 & 0.23 & 0.63 & 0.14 & 0.95 & 1.21 & 0.47 & 0.82 & 1.22 & 0.56 & $\| G$ \\
\hline Glyma11g04130 & JAZ1 & -2.06 & -0.67 & 0.67 & 0.56 & 0.63 & 2.15 & 2.21 & 1.49 & 1.88 & 2.01 & 1.10 & $\mathrm{IIG} / \mathrm{ClG}$ \\
\hline Glyma13g17180 & JAZ1 & -1.09 & -0.40 & 0.45 & 0.48 & 0.71 & 1.61 & 1.93 & 1.27 & 1.50 & 1.84 & 1.17 & $\mathrm{IIG} / \mathrm{ClG}$ \\
\hline Glyma15g19840 & JAZ1 & -0.98 & -0.37 & 0.33 & 0.28 & 0.28 & 1.00 & 1.09 & 0.39 & 0.78 & 1.15 & 0.53 & $\| G$ \\
\hline Glyma17g05540 & JAZ1 & -2.02 & -0.93 & 0.24 & 0.45 & 0.45 & 1.59 & 1.46 & 0.66 & 1.54 & 1.42 & 0.57 & $\mathrm{IIG} / \mathrm{ClG}$ \\
\hline Glyma07g04630 & JAZ6 & -0.83 & -0.73 & 0.14 & 0.20 & 0.41 & 1.02 & 0.92 & 0.82 & 1.08 & 1.24 & 0.57 & IIG \\
\hline Glyma16g01220 & JAZ6 & -0.91 & -0.43 & 0.41 & 0.42 & 0.65 & 1.45 & 1.37 & 1.05 & 1.40 & 1.32 & 0.84 & $\| G$ \\
\hline Glyma05g27280 & JAZ8 & -1.59 & -1.72 & 2.40 & 0.95 & 0.32 & 3.85 & 2.08 & 1.68 & 3.05 & 3.16 & 2.73 & $\| G$ \\
\hline Glyma08g10220 & JAZ8 & 0.09 & -0.55 & 1.22 & 0.94 & 0.97 & 3.35 & 3.01 & 3.12 & 3.43 & 4.12 & 2.94 & $\| G$ \\
\hline Glyma13g29070 & JAZ8 & -3.00 & -1.08 & 0.21 & 0.52 & 0.50 & 2.13 & 2.27 & 1.70 & 2.57 & 1.94 & 1.28 & $\mathrm{IIG} / \mathrm{CIG}$ \\
\hline Glyma13g17640 & JAZ12 & -0.82 & -0.19 & 0.63 & 0.27 & 0.61 & 1.65 & 0.35 & 0.86 & 1.50 & 1.03 & 0.21 & $\| G$ \\
\hline Glyma17g04850 & JAZ12 & -1.05 & -0.59 & 0.05 & -0.49 & 0.42 & 1.04 & -0.49 & 0.44 & 0.07 & 0.27 & -0.23 & CIG \\
\hline Glyma07g06370 & JAR1 & 1.11 & 0.46 & 0.21 & 0.68 & 0.53 & 0.42 & 0.10 & 0.47 & -0.06 & 0.22 & 0.39 & $\mathrm{CIG}$ \\
\hline Glyma20g21780 & ETR2 & -0.56 & -0.98 & -0.41 & -0.27 & -1.06 & -0.69 & -0.71 & -0.72 & -0.84 & -1.00 & -0.27 & $\| G$ \\
\hline Glyma20g34420 & ETR2 & 1.33 & 0.45 & 0.89 & 0.84 & 0.34 & 0.08 & 0.41 & 0.73 & -0.34 & -0.29 & 0.40 & CIG \\
\hline Glyma03g41220 & EIN4 & -0.47 & -0.68 & -0.90 & -1.13 & -1.37 & -2.65 & -1.86 & -1.88 & -2.05 & -2.40 & -1.04 & $\| G$ \\
\hline Glyma19g43840 & EIN4 & -0.41 & -0.19 & -0.78 & 0.00 & -0.40 & -0.94 & -0.60 & -0.33 & -1.38 & -1.44 & -0.18 & $\| G$ \\
\hline Glyma04g07110 & EBF1 & 0.79 & -0.08 & -0.16 & 0.62 & -0.91 & -0.77 & -0.68 & -0.94 & -1.39 & -0.79 & -0.56 & IIG \\
\hline Glyma04g20330 & EBF1 & 2.68 & 0.79 & 0.29 & 1.04 & -0.26 & 0.16 & 0.52 & 0.35 & -0.51 & -0.08 & 0.98 & $\mathrm{CIG}$ \\
\hline Glyma06g07200 & EBF1 & 1.18 & -0.02 & -0.27 & 0.88 & -0.79 & -0.53 & -0.58 & -0.75 & -1.29 & -0.88 & -0.22 & $\mathrm{IIG} / \mathrm{ClG}$ \\
\hline Glyma13g23510 & EBF1 & 1.71 & 0.37 & 0.38 & 0.94 & -0.32 & 0.04 & 0.13 & -0.49 & -0.79 & -0.38 & 0.57 & $\mathrm{CIG}$ \\
\hline Glyma17g31940 & EBF1 & 1.32 & 0.01 & -0.24 & 0.95 & -0.98 & -0.95 & -0.83 & -0.83 & -1.56 & -0.99 & -0.36 & $\mathrm{IIG} / \mathrm{ClG}$ \\
\hline Glyma05g24770 & BAK1 & 0.19 & 1.14 & 2.02 & 2.40 & 2.03 & 2.88 & 3.19 & 2.14 & 2.84 & 3.40 & 2.27 & $\| G$ \\
\hline Glyma08g19270 & BAK1 & 0.75 & 0.67 & 0.94 & 0.76 & 0.68 & 0.75 & 1.22 & 1.16 & 0.92 & 0.85 & 0.76 & IIG \\
\hline Glyma15g05730 & BAK1 & 0.85 & 0.82 & 0.75 & 0.84 & 0.77 & 0.95 & 1.07 & 1.43 & 0.99 & 0.98 & 0.93 & IIG \\
\hline
\end{tabular}

The ET signaling pathway is generally considered to work cooperatively with JA in response to necrotrophic pathogens [8,22]. In Arabidopsis, ET receptors ETR2 [43] and EIN4 [44] were both negative regulators of ET signaling, and an ubiquitin/proteasome pathway for the degradation of EIN3 was mediated by an F-box protein, $E B F 1$, to negatively regulate ET responses [23]. We found that one homolog of ETR2 and two homologs of EIN4 were down-regulated in NILs, while another homolog of ETR2 was up-regulated in Williams. In addition to ETR2 and EIN4, we identified five $E B F 1$ homologs that were down-regulated in all NILs and upregulated in Williams (Figure 5), suggesting that the ET signaling response to the pathogen was repressed during compatible interactions but activated during incompatible interactions.

In addition to the three major phytohormones described above, BR have also been found to enhance disease resistance against viral and bacterial pathogens in tobacco and rice [45]. BAK1 is a crucial component of BR signaling, and can interact with LRR-RLK (leucinerich repeat receptor-like protein kinase) genes such as 
FLS2 to promote their function in plant defense responses [46]. As shown in Figure 5, three $B A K 1$ homologs were identified as IIGs and all were up-regulated upon inoculation with the pathogen, suggesting a positive role of $\mathrm{BR}$ signaling in defense response to $P$. sojae.

\section{Discussion}

The nature of distinction in molecular immune response among NILs

One of the most striking observations in this study was the distinction in defense response to inoculation with $P$. sojae among the ten resistant NILs as revealed by comparative transcriptomic analysis. This was reflected not only by the level of variation in the number of IIGs (ranging from 300 to 3073) detected in individual NILs, but also by the relative small numbers of up-regulated (16) and down-regulated (7) IIGs shared by all NILs (Table 3). In general, different Rps genes recognize distinct Avr genes in a P. sojae population [47], but the Avr determinants of different Rps genes in a same isolate of the pathogen (i.e., race 1 in this study) may not be distinct. One hypothesis that may explain the observed variations of DEGs among different NILs may be differential timing and robustness in defense responses and signaling mediated by different Rps genes/alleles [48]. Although the timing of gene expression mediated by different $R$ genes in response to the same pathogen has not been characterized in plants, dramatic and rapid changes in gene expression after inoculation with, or following infection by a pathogen, have been observed [24,49]. A recent study demonstrated that $\sim 5 \%$ of DEGs were shared by soybean lines resistant to bacterial leaf pustule at 6 and 12 hpi with Xanthomonas axonopodis [33]. In this study, the majority ( $>95 \%)$ of DEGs showed less than an eight-fold difference in expression levels upon inoculation with the pathogen when given the same cutoff time for each of the 11 lines. Such a low level of expressional changes and uniformed cutoff may have affected the detectability of shared DEGs among different lines. In addition, the NILs remain 1-2\% genomic difference (mostly surrounding the Rps loci) from each other, which may have affected expression patterns of a small proportion of genes. Furthermore, inoculated regions of seedling stems, near which layers of cells respond to the pathogen, vary in size among different plants and NILs. Such a variation may affect effective identification of DEGs. Therefore, the numbers of DEGs, and particularly IIGs specific to each NIL could be over-estimated. Nevertheless, the DEGs in each line were detected pre-and post-inoculation with the same pathogen, and thus the influence of genomic difference surrounding Rps genes on our pipeline for detection of DEGs triggered by the pathogen may be minimal.

It is documented that resistance genes recognize pathogens either directly or indirectly by guarding a protein or using a decoy [13]. In Arabidopsis, RIN4 is 'guarded' by resistance genes $R P M 1$ and $R P S 2$, which trigger the immune response $[14,15]$. In soybean, two of four RIN4homologous genes appear to function as a heteromeric complex in mediating RPG1-B and RPM1-derived resistance to Pseudomonas syringae, and silencing GmRIN4a or GmRIN4b in rpg1-b plants enhances basal resistance to virulent strains of $P$. syringae and the oomycete $P$. sojae [50]. In our study, a single RIN4 homolog was found to be up-regulated in C-III NILs, but not in NILs within C-I and C-II. If this RIN4 homolog did encode the "guard" protein that was recognized by the Rps genes in the C-III NILs, then the relatively low numbers of IIGs identified among NILs in C-I and C-II, as compared with the C-III NILs, may be explained by the distinct "recognition" processes needed for triggering the immune response, which may result in variations in speed, timing, and magnitude of molecular responses to the pathogen $[48,51]$.

\section{Do patterns in transcriptomics distinguish Rps genes from alleles?}

An allele is defined as an alternative form of the same gene that is located at a specific position on a specific chromosome. In general, different alleles of the same gene are determined by genetic tests for allelism. However, due to the complexity of phenotypes, such as variation in the proportion of surviving plants of a pure NIL that contains an Rps gene, as observed in this study, such a test may not be effective in determining whether the resistance carried by two individual lines is controlled by different alleles or different genes. Moreover, many NBS-LRR gene models predicted in the Williams82 genome are clustered in a small number of genomic regions $[32,52]$, which makes it more difficult to distinguish genes resistant to the same pathogen by classic genetic analysis, especially if they are physically adjacent or closely linked to each other.

The Rps genes/alleles in the 10 NILs have been previously mapped to three genomic regions: Rps1- $a$, Rps1- $b$, Rps1-c, and Rps1- $k$ on chromosome 3 [6,53,54], Rps3- $a$, Rps3-b and Rps3-c on chromosome 13 [1,54,55] and Rps4, Rps5 and Rps6 on chromosome 18 [1,54,55]. However, because limited numbers of molecular markers were available and used when these genes/alleles were identified, the genetic distances between genes or alleles mapped in the same chromosomal regions have not been determined. Actually, the alleles at the Rps1 and Rps3 loci were simply designated based on their disease reaction to a set of $P$. sojae races. As these three regions are highly enriched with NBS-LRR genes, according to the soybean reference genome, it is possible that some designated alleles of the same gene may be located at different loci. 
As different alleles of the same gene are generally more identical to each other than to other genes, people are inclined to speculate that the defense response mediated by different alleles of a same gene may be more similar than those mediated by different genes. At first glance, this hypothesis seems to be supported by the observation that the NILs with Rps3- $a$ and Rps3- $b$ exhibited similar patterns of transcriptional changes upon inoculation with $P$. sojae, as did the NILs with Rps1-b and Rps1-c (Figure 2). However, the pattern of transcriptional changes mediated by $R p s 3-c$ was found to be most similar to those mediated by Rps4, Rps5, and Rps6. Thus, the patterns of transcriptomic changes do not appear to predict genetic similarity and difference among these Rps genes and alleles, although we could not rule out the possibility that Rps3-c and Rps1- $a$ are non-allelic to the Rps3 and Rps1 loci, respectively.

\section{An integrated picture of molecular responses to $P$. sojae}

Based on analysis of putative defense-related genes identified in this study and the patterns of their expressions upon inoculation with the pathogen, we propose a hypothesis that four major phytohormone signaling pathways are involved in defense responses (Figure 6A). During incompatible interaction, the Rps protein recognizes the avirulence effector of $P$. sojae, initiating signaling transduction that involves the SA, JA, ET and BR pathways. The SA, ET, and BR pathways are activated, whereas the JA pathway is suppressed. Although the specific functions of individual genes encoding TFs belonging to the WRKY family, as well as those genes for reactive oxygen species (ROS) and MAP kinase (MAPK) cascades remain to be elucidated, it is apparent that some of the genes belonging to these families play pivotal roles in defense signaling transduction. During compatible interaction, the avirulence effector of $P$. sojae induces a set of downstream responses that lead to disease development (Figure 6B). The signatures of the susceptible responses would include up-regulation of the JA pathway, suppression of ET pathway and with no significant changes in SA and BR pathways. It seems possible that the defense responses might be delayed in the compatible interaction due to insufficient activation of the expression of genes related to ROS and phytoalexin biosynthesis, as well as MAPK signaling. Together, these data gained from a unique set of soybean NILs provide a comprehensive picture regarding the molecular mechanisms underlying incompatible and compatible interaction between soybean and $P$. sojae, which shed light on the nature and timing of molecular responses mediated by individual Rps genes.

\section{Conclusions}

Comparative transcriptomic analysis of ten resistant NILs and susceptible recurrent line Williams with and without inoculation allowed us to identify DEGs associated with defense responses to $P$. sojae that were unique in each NIL and common among all these NILs, and thus depicted functional "fingerprints" of individual Rps-mediated resistance. Further analysis revealed multiple branches of putative ET, JA, ROS, and MAPK regulatory networks underlying the defense responses. Such responses exhibited dramatic variations among the soybean NILs containing distinct Rps genes/alleles. We propose different timing of individual Rps-mediated defense signaling to the same pathogen may be largely responsible for such variations.
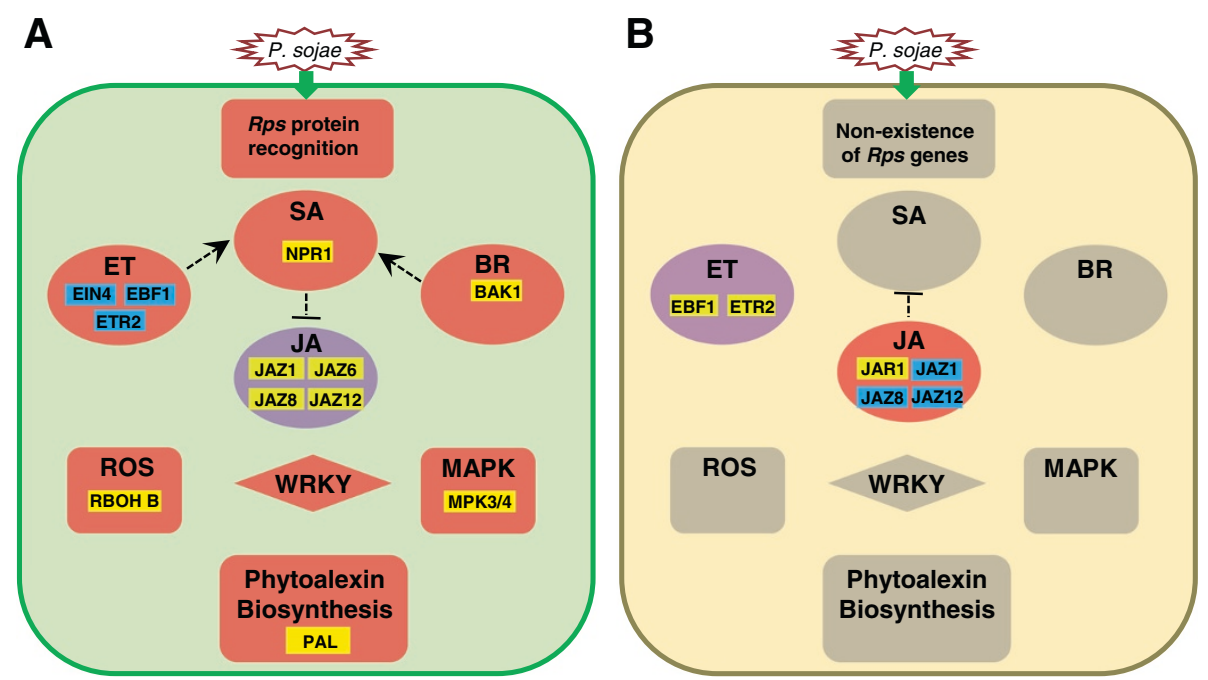

Figure 6 An integrated picture for gene network of interactions between soybean and race1 of Phytophthora sojae. A: Incompatible interaction. B: Compatible interaction. Red = up-regulation of a pathway. Purple= down-regulation of a pathway. Grey = no significant change in pathway. Yellow = up-regulation of genes. Blue = down-regulation of genes. Dotted lines indicate interactions of pathways inferred from Arabidopsis studies. 


\section{Methods}

\section{Plant materials and isolates of $P$. sojae}

The susceptible cultivar Williams and its ten NILs, each containing a unique Rps gene/allele, were used this study (Additional file 1). Each NIL was generated by backcrossing the donor for at least five generations with Williams. For inoculation tests, the isolate $p m g(1)-3$ (pathotype race1) of $P$. sojae was used.

\section{Treatment of soybean materials}

Seeds of each soybean line were planted in sterilized sand in $10.1 \mathrm{~cm}$ clay pots and placed in a greenhouse. Approximately seven days after planting, at the VC growth stage (unifoliate leave fully expanded), the seedlings for each line were separated into four groups, each containing $\sim 20$ seedlings. Two groups were challenged with $P$. sojae using a standard hypototyl inoculation method [56], while the other two groups were mock inoculated (wounded) without the pathogen. At $24 \mathrm{hpi}$, stems were harvested from one set of inoculated and one set of wounded seedlings by excising 2 to $3 \mathrm{~cm}$ across the wounded site and storing immediately in liquid nitrogen. The seedlings in the remaining two groups were kept for evaluating symptom development, which was recorded 7 dpi. Experiments were performed a total of three times.

\section{RNA sequencing and analysis}

Total RNAs were isolated using the RNeasy Plant Mini Kit (Qiagen Inc., Valencia, CA) according to the manufacturer's instructions in conjunction with DNase. The quality of total RNA was determined using a Nanodrop spectrometer (Thermo Fisher Scientific Inc., Wilmington, DE) and 1\% formaldehyde gel electrophoresis. Total RNA samples were then sent to the Genomics Center at Purdue University for sequencing and 101 bp paired-end reads were generated with the Illumina HiScanSQ system. Since samples were a mixture of RNA from both soybean and $P$. sojae, to exclude the effect of $P$. sojae sequences, all reads that aligned to the $P$. sojae genome sequence were eliminated [57]. All remaining reads were then aligned to G. $\max$ reference genome (v8.0, http:// www.phytozome.net) using TopHat software [58] with parameters set to allow only one mismatch, and 30 and $100,000 \mathrm{bp}$ of the minimum and maximum intron length based on the current gene annotation. Moreover, only the uniquely mapped reads or fragments were kept for further analyses. The raw count of each gene was calculated by HTSeq (http://www-huber.embl.de/users/anders/HTSeq) with the "intersection_nonempty" mode, and preceded to edgeR package [59] in the R-Bioconductor tools.

To detect variability, we estimated the over dispersion from 40 highly confident soybean housekeeping genes collected from previously reported papers (Additional file 10) [24,33,60-63], considering different soybean lines as replicates of control and inoculation groups. The data were then modeled using a Negative Binomial model in edgeR to identify differentially expressed genes after inoculation using the dispersion estimated from the housekeeping genes as a common dispersion. Any genes with coverage read count less than one count per million of two paired samples were removed in later analyses. Differential expression between the inoculation group and the mock-inoculation group for each line was tested for false discovery rate (FDR) that was controlled at 0.05 [64] using edgeR package [59].

\section{Hierarchical cluster and heatmap analysis}

Pvclust and hclust were performed using the $\log _{2} \mathrm{FC}$ of the 5,806 IIGs with default parameters using distance method "correlation" for complete linkage clustering analysis. Pvclust provides two types of $p$-values to assess the uncertainty for each cluster: approximately unbiased (AU) p-value and bootstrap probability (BP) value, via multi-scale bootstrap re-sampling [35]. The heatmap representing the expression intensity and direction was drawn using pheatmap $R$ package with the distance method "euclidean" for both rows and columns [65].

\section{Gene Ontology, heatmap and homologous gene functional analysis}

Annotations of GO terms were obtained from the AgriGO website based on the G. max model [66], and GO biological process categories were used. These terms were manually classified into six broad functional groups, 'response to biotic or abiotic stress', 'biological regulation,' 'growth and development', 'transport', 'metabolism', and 'miscellaneous', while genes without GO annotations were grouped as 'unclassified'. For genes with multiple GO categories, only one category was selected based on priority. Homologous genes were searched against annotated Arabidopsis gene protein database (The Arabidopsis Information Resouces, www.arabidopsis.org) using BLASTP to verify gene functions manually.

\section{Quantitative real-time PCR analysis}

The qRT-PCR was carried out using StepOnePlus ${ }^{\mathrm{Tm}}$ Real Time PCR system (Applied Biosystems). The RNA samples used as templates for qRT-PCR were the same as those used for RNA-Seq. The cons 4 gene [60] was used as internal control for normalization of qRT-PCR data. For each gene, three experimental replicates were performed. Pearson correlations were calculated between RNA-Seq and qRT-PCR methods using six out of seven randomly selected genes for Log2 fold change of inoculated and mock-inoculated treatments in each soybean line (Additional file 3). 


\section{Availability of supporting data}

The transcriptome sequences presented in this study have been deposited in NCBI Gene Expression Omnibus under accession numbers GSE48524.

\section{Additional files}

\section{Additional file 1: Breeding pedigree of soybean NILs used in this study. \\ Additional file 2: Reactions of soybean NILs to Phytophthora sojae. \\ Additional file 3: List of primers used in $\mathrm{qPCR}$. \\ Additional file 4: Comparison in expression of six soybean genes in Williams and 10 NILs, each containing a single Rps gene, as determined by RNA-Seq analysis or qRT-PCR. $Y$ axis indicates differen- tial expression of selected genes for each soybean line. $X$ axis indicates selected genes used for qRT-PCR. These genes are Glyma02g47940 (1), Glyma04g20330 (2), Glyma05g24770 (3), Glyma07g07270 (4), Glyma09g37290 (5), Glyma10g44170 (6), and Glyma11g04130 (7). Pearson's corrleation coefficient (r).}

Additional file 5: Heatmap (hcluster) analysis of 5,806 IIGs for 10 soybean NILs, each containing a single Rps gene. The values used to draw heatmap is $\log _{2}$ (fold change) of expression level of post inoculation to mock inoculation.

Additional file 6: GO analysis of DEGs in different categories.

Additional file 7: Gene ontology categories for IIGs identified in soybean NILs, each containing a single Rps gene. Left bar = upregulated genes. Right bar $=$ down-regulated genes

Additional file 8: Comparison of differentially expressed transcription factors (DETFs) between Williams and 10 NILs each containing a single Rps gene. A: up-regulated DETFs. B: downregulated DETFs. Red and purple represent the number of DETFs specific to individual Rps genes and collectively referred to as incompatible interaction transcription factors. Brown and dark purple represent the number of DETFs shared by an individual NIL and Williams. Green and light blue represent the number of DETFs specifically expressed in Williams when compared to a specific NIL. The central green and light blue circles represent the common proportion of Williams-specific DETFs that are collectively referred to as compatible interaction transcription factors.

Additional file 9: Counts of transcription factors (TFs) in different groups.

Additional file 10: Housekeeping genes selected in this study.

\section{Competing interests}

The authors declare that they have no competing interests.

\section{Authors' contributions}

TJH and JM designed the research; FL, MZ, RWD, TJH, and JM interpreted the data; FL, MZ, DDB, JP, LS, YL, BZ, ZT performed the research; FL, MZ, RWD, $\mathrm{TJH}$, and JM drafted and revised the manuscript; FL, MZ, DDB, JP, LS, YL, BZ, $Z T, R W D, T J H$, and JM approved the submission of the version. All authors read and approved the final manuscript.

\section{Acknowledgements}

This work was mainly supported by soybean checkoff funds from the Indiana Soybean Alliance (Grant No. 205267), and partially supported by "Partnership for Research \& Education in Plant Breeding and Genetics" program funded by the U.S. Department of Agriculture's National Institute of Food and Agriculture, and corporate partners Ag Alumni Seed, AgReliant Genetics, Beck's Hybrids, ConAgraFoods, Dow AgroSciences, Indiana Crop Improvement Association and Pioneer Hi-Bred International. We also thank Brittany Radke, Tomara J. Fleury and Brian Foss for their help in inoculation and preparation of inoculum.

\section{Author details}

'Department of Agronomy, Purdue University, West Lafayette, IN 47907, USA. 2 Department of Mathematics, University of Wisconsin - La Crosse, La Crosse, WI 54601, USA. ${ }^{3}$ Department of Statistics, Purdue University, West Lafayette, IN 47907, USA. ${ }^{4}$ Department of Botany and Plant Pathology, Purdue University, West Lafayette, IN 47907, USA. ' USDA-ARS Crop Production and Pest Control Research Unit, Purdue University, West Lafayette, IN 47907, USA.

Received: 26 October 2013 Accepted: 7 January 2014

Published: 10 January 2014

\section{References}

1. Sandhu D, Schallock KG, Rivera-Velez N, Lundeen P, Cianzio S, Bhattacharyya MK: Soybean Phytophthora resistance gene Rps8 maps closely to the Rps3 region. J Hered 2005, 96:536-541.

2. Dorrance AE, Mills D, Robertson AE, Draper MA, Giesler L, Tenuta A: Phytophthora root and stem rot of soybean. The Plant Health Instructor 2007. 10.1094/PHI-I-2007-0830-07.

3. Tyler BM: Phytophthora sojae: root rot pathogen of soybean and model oomycete. Mol Plant Pathol 2007, 8:1-8.

4. Sugimoto T, Kato M, Yoshida S, Matsumoto I, Kobayashi T, Kaga A, Hajika M, Yamamoto R, Watanabe K, Aino M, et al: Pathogenic diversity of Phytophthora sojae and breeding strategies to develop Phytophthoraresistant soybeans. Breeding Sci 2012, 61:511-522.

5. Lin F, Zhao M, Ping J, Johnson A, Zhang B, Abney TS, Hughes TJ, Ma J: Molecular mapping of two genes conferring resistance to Phytophthora sojae in a soybean landrace PI 567139B. Theor Appl Genet 2013, 126:2177-2185

6. Gao H, Narayanan NN, Ellison L, Hattacharyya MK: Two classes of highly similar coiled coil-nucleotide binding-leucine rich repeat genes isolated from the Rps 1-k locus encode Phytophthora resistance in soybean. $\mathrm{Mol}$ Plant-Microbe Interact 2005, 18:1035-1045.

7. Dangl $\mathrm{J}$, Jones JDG: Plant pathogens and integrated defence responses to infection. Nature 2001, 411:826-833.

8. Jones JDG, Dangl JL: The plant immune system. Nature 2006, 444:323-329.

9. Dodds PN, Rathjen JP: Plant immunity: towards an integrated view of plant-pathogen interactions. Nat Rev Genet 2010, 11:539-548.

10. Fliegmann J, Mithofer A, Wanner G, Ebel J: An ancient enzyme domain hidden in the putative beta-glucan elicitor receptor of soybean may play an active part in the perception of pathogen-associated molecular patterns during broad host resistance. J Biol Chem 2004, 279:1132-1140.

11. Dodds PN, Lawrence GJ, Catanzariti AM, Ayliffe MA, Ellis JG: The Melampsora lini AvrL567 avirulence genes are expressed in haustoria and their products are recognized inside plant cells. Plant Cell 2004 16:755-768

12. Van der Biezen EA, Jones JDG: Plant disease-resistance proteins and the gene-for-gene concept. Trends Biochem Sci 1998, 23:454-456.

13. Van der Hoorn RAL, Kamoun S: From guard to decoy: a new model for perception of plant pathogen effectors. Plant Cell 2008, 20:2009-2017.

14. Belkhadir Y, Nimchuk Z, Hubert DA, Mackey D, Dangl JL: Arabidopsis RIN4 negatively regulates disease resistance mediated by RPS2 and RPM1 downstream or independent of the NDR1 signal modulator and is not required for the virulence functions of bacterial type III effectors AvrRpt2 or AvrRpm1. Plant Cell 2004, 16:2822-2835

15. Mackey D, Belkhadir Y, Alonso JM, Ecker JR, Dangl JL: Arabidopsis RIN4 is a target of the type III virulence effector AvrRpt2 and modulates RPS2-mediated resistance. Cell 2003, 112:379-389.

16. Glazebrook J, Rogers EE, Ausubel FM: Isolation of Arabidopsis mutants with enhanced disease susceptibility by direct screening. Genetics 1996, 143:973-982.

17. Aarts N, Metz M, Holub E, Staskawicz BJ, Daniels MJ, Parker JE: Different requirements for EDS1 and NDR1 by disease resistance genes define at least two R gene-mediated signaling pathways in Arabidopsis. Proc Nat Acad Sci USA 1998, 95:10306-10311.

18. Zhou N, Tootle TL, Tsui F, Klessig DF, Glazebrook J: PAD4 functions upstream from salicylic acid to control defense responses in Arabidopsis. Plant Cell 1998, 10:1021-1030

19. Falk A, Feys BJ, Frost LN, Jones JDG, Daniels MJ, Parker JE: EDS1, an essential component of $R$ gene-mediated disease resistance in Arabidopsis has homology to eukaryotic lipases. Proc Nat Acad Sci USA 1999, 96:3292-3297. 
20. Wiermer M, Feys BJ, Parker JE: Plant immunity: the EDS1 regulatory node. Curr Opin Plant Biol 2005, 8:383-389.

21. Bhattacharjee S, Halane MK, Kim SH, Gassmann W: Pathogen effectors target Arabidopsis EDS1 and alter its interactions with immune regulators. Science 2011, 334:1405-1408.

22. Glazebrook J: Contrasting mechanisms of defense against biotrophic and necrotrophic pathogens. Annu Rev Phytopathol 2005, 43:205-227.

23. Robert-Seilaniantz A, Grant $M$, Jones JDG: Hormone crosstalk in plant disease and defense: more than just jasmonate-salicylate antagonism. Annu Rev Phytopathol 2011, 49:317-343.

24. Moy P, Qutob D, Chapman BP, Atkinson I, Gijzen M: Patterns of gene expression upon infection of soybean plants by Phytophthora sojae. Mol Plant-Microbe Interact 2004, 17:1051-1062.

25. Sandhu D, Tasma IM, Frasch R, Bhattacharyya MK: Systemic acquired resistance in soybean is regulated by two proteins, Orthologous to Arabidopsis NPR1. BMC Plant Biol 2009, 9:105.

26. Sugano S, Sugimoto T, Takatsuji $H$, Jiang $C$ J: Induction of resistance to Phytophthora sojae in soyabean (Glycine max) by salicylic acid and ethylene. Plant Pathol 2013, 62:1048-1056.

27. Narayanan NN, Grosic S, Tasma IM, Grant D, Shoemaker R, Bhattacharyya M Identification of candidate signaling genes including regulators of chromosome condensation 1 protein family differentially expressed in the soybean-Phytophthora sojae interaction. Theor Appl Genet 2009, 118:399-412.

28. Ward EWB, Cahill DM, Bhattacharyya M: Abscisic acid suppression of phenylalanine ammonialyase activity and mRNA, and resistance of soybeans to Phytophthora megasperma f. sp. glycinea. Plant Physiol 1989 91:23-27.

29. McDonald KL, Cahill DM: Influence of abscisic acid and the abscisic acid biosynthesis inhibitor, norflurazon, on interactions between Phytophthora sojae and soybean (Glycine max). Eur J Plant Pathol 1999, 105:651-658.

30. Zhou L, Mideros SX, Bao L, Hanlon R, Arredondo FD, Tripathy S, Krampis K, Jerauld A, Evans C, St Martin SK, et al: Infection and genotype remodel the entire soybean transcriptome. BMC Genomics 2009, 10:49.

31. Zhang Y, Zhao J, Xiang Y, Bian X, Zuo Q, Shen Q, Gai J, Xing H: Proteomics study of changes in soybean lines resistant and sensitive to Phytophthora sojae. Proteome Sci 2011, 9:52.

32. Schmutz J, Cannon SB, Schlueter J, Ma JX, Mitros T, Nelson W, Hyten DL, Song QJ, Thelen JJ, Cheng JL, et al: Genome sequence of the palaeopolyploid soybean. Nature 2010, 463:178-183.

33. Kim KH, Kang YJ, Kim DH, Yoon MY, Moon JK, Kim MY, Van K, Lee SH: RNASeq analysis of a soybean near-isogenic line carrying bacterial leaf pustule-resistant and -susceptible alleles. DNA Res 2011, 18:483-497.

34. Dorrance AE, Berry SA, Anderson TR, Meharg C: Isolation, storage, pathotype characterization, and evaluation of resistance for Phytophthora sojae in soybean. Plant Health Progress 2008. 10.1094/PHP2008-0118-01-DG.

35. Suzuki R, Shimodaira H: Pvclust: an R package for assessing the uncertainty in hierarchical clustering. Bioinformatics 2006, 22:1540-1542.

36. Eisen MB, Spellman PT, Brown PO, Botstein D: Cluster analysis and display of genome-wide expression patterns. Proc Nat Acad Sci USA 1998, 95:14863-14868.

37. Wang Z, Libault M, Joshi T, Valliyodan B, Nguyen HT, Xu D, Stacey G, Cheng J: SoyDB: a knowledge database of soybean transcription factors. $B M C$ Plant Biol 2010, 10:14.

38. Dong X: NPR1, all things considered. Curr Opin Plant Biol 2004, 7:547-552

39. Gfeller A, Liechti R, Farmer EE: Arabidopsis jasmonate signaling pathway. Science 2010, 3:1-2.

40. Pauwels L, Goossens A: The JAZ proteins: a crucial interface in the jasmonate signaling cascade. Plant Cell 2011, 23:3089-3100.

41. Staswick PE, Tiryaki I, Rowe ML: Jasmonate response locus JAR1 and several related Arabidopsis genes encode enzymes of the firefly luciferase superfamily that show activity on jasmonic, salicylic, and indole-3-acetic acids in an assay for adenylation. Plant Cell 2002, 14:1405-1415.

42. Staswick PE, Tiryaki I: The oxylipin signal jasmonic acid is activated by an enzyme that conjugates it to isoleucine in Arabidopsis. Plant Cell 2004, 16:2117-2127.

43. Sakai H, Hua J, Chen QG, Chang C, Medrano LJ, Bleecker AB, Meyerowitz EM: ETR2 is an ETR1-like gene involved in ethylene signaling in Arabidopsis. Proc Nat Acad Sci USA 1998, 95:5812-5817.
44. Hua J, Sakai H, Nourizadeh S, Chen QG, Bleecker AB, Ecker JR, Meyerowitz EM: EIN4 and ERS2 are members of the putative ethylene receptor gene family in Arabidopsis. Plant Cell 1998, 10:1321-1332.

45. Nakashita H, Yasuda M, Nitta T, Asami T, Fujioka S, Arai Y, Sekimata K Takatsuto S, Yamaguchi I, Yoshida S: Brassinosteroid functions in a broad range of disease resistance in tobacco and rice. Plant J 2003, 33:887-898.

46. Clouse SD: Brassinosteroid signal transduction: from receptor kinase activation to transcriptional networks regulating plant development. Plant Cell 2011, 23:1219-1230.

47. May KJ, Whisson SC, Zwart RS, Searle IR, Irwin JAG, Maclean DJ, Carroll BJ, Drenth A: Inheritance and mapping of 11 avirulence genes in Phytophthora sojae. Fungal Genet Biol 2002, 37:1-12.

48. Tao Y, Xie Z, Chen W, Glazebrook J, Chang HS, Han B, Zhu T, Zou G, Katagiri F: Quantitative nature of Arabidopsis responses during compatible and incompatible interactions with the bacterial pathogen Pseudomonas syringae. Plant Cell 2003, 15:317-330.

49. Singh KB, Foley RC, Onate-Sanchez L: Transcription factors in plant defense and stress responses. Curr Opin Plant Biol 2002, 5:430-436.

50. Selote D, Kachroo A: RPG1-B-derived resistance to AvrB-expressing Pseudomonas syringae requires RIN4-like proteins in soybean. Plant Physiol 2010, 53:1199-1211.

51. Dickman MB, de Figueiredo P: Comparative pathobiology of fungal pathogens of plants and animals. Plos Pathog 2011, 7:e1002324.

52. McHale LK, Haun WJ, Xu WW, Bhaskar PB, Anderson JE, Hyten DL, Gerhard DJ, Jeddeloh JA, Stupar RM: Structural variants in the soybean genome localize to clusters of biotic stress-response genes. Plant Physiol 2012, 159:1295-1308.

53. Weng C, Yu K, Anderson TR, Poysa V: Mapping genes conferring resistance to Phytophthora root rot of soybean, Rps1a and Rps7. J Hered 2001, 92:442-446.

54. Demirbas A, Rector BG, Lohnes DG, Fioritto RJ, Graef GL, Cregan PB, Shoemaker RC, Specht JE: Simple sequence repeat markers linked to the soybean Rps genes for Phytophthora resistance. Crop Sci 2001, 41:1220-1227

55. Gordon SG, Martin SKS, Dorrance AE: Rps8 maps to a resistance gene rich region on soybean molecular linkage group F. Crop Sci 2006, 46:168-173.

56. Abney TS, Melgar JC, Richards TL, Scott DH, Grogan J, Young J: New races of Phytophthora sojae with Rps1-d virulence. Plant Dis 1997, 81:653-655.

57. Tyler BM, Tripathy S, Zhang X, Dehal P, Jiang RHY, Aerts A, Arredondo FD, Baxter $L$, Bensasson D, Beynon $J$, et al: Phytophthora genome sequences uncover evolutionary origins and mechanisms of pathogenesis. Science 2006, 313:1261-1266.

58. Trapnell C, Pachter L, Salzberg SL: TopHat: discovering splice junctions with RNA-seq. Bioinformatics 2009, 25:1105-1111.

59. Robinson MD, McCarthy DJ, Smyth GK: edgeR: a Bioconductor package for differential expression analysis of digital gene expression data. Bioinformatics 2010, 26:139-140.

60. Libault M, Thibivilliers S, Bilgin DD, Radwan O, Benitez M, Clough SJ, Stacey $\mathrm{G}$ : Identification of four soybean reference genes for gene expression normalization. Plant Genome 2008, 1:44-54.

61. Jian B, Liu B, Bi Y, Hou W, Wu C, Han T: Validation of internal control for gene expression study in soybean by quantitative real-time PCR. BMC Mol Biol 2008, 9:59.

62. Hu R, Fan C, Li H, Zhang Q, Fu YF: Evaluation of putative reference genes for gene expression normalization in soybean by quantitative real-time RT-PCR. BMC Mol Biol 2009, 10:93.

63. Stolf-Moreira R, Lemos EGDM, Abdelnoor RV, Beneventi MA, Rolla AAP, Pereira SD, de Oliveira MCN, Nepomuceno AL, Marcelino-Guimaraes FC: Identification of reference genes for expression analysis by real-time quantitative PCR in drought-stressed soybean. Pesqui Agropecu Bras 2011, 46:58-65.

64. Benjamini $Y$, Hochberg $Y$ : Controlling the false discovery rate - a practical and powerful approach to multiple testing. J R Stat SoC B 1995, 57:289-300. 65. Kolde R: pheatmap: pretty heatmap. R package version 0612012.

66. Du Z, Zhou X, Ling Y, Zhang ZH, Su Z: agriGO: a GO analysis toolkit for the agricultural community. Nucleic Acids Res 2010, 38:W64-W70.

doi:10.1186/1471-2164-15-18

Cite this article as: Lin et al:: Molecular response to the pathogen Phytophthora sojae among ten soybean near isogenic lines revealed by comparative transcriptomics. BMC Genomics 2014 15:18. 